\title{
An Effective Computation Method Based on Field Velocity Approach for Acceleration Derivative
}

\author{
Jun Yang, Yu Qian \\ Flight Technology College, Civil Aviation Flight University of China, Guanghan, China \\ yangcafc@163.com, qianyucafuc@hotmail.com
}

\begin{abstract}
A novel method of calculating static derivatives and acceleration derivatives using Computational Fluid Dynamics is presented. This method uses field velocity approach to simulate the unsteady forced-plunging flow field over the aircraft without updating the field grids during each time step. This provides an improvement in computational efficiency over similar precision without loss of generality. Finally, to verify the present method, the Basic Finner missile as the benchmark example was taken to calculate its acceleration derivatives at various Mach number. Results are in fairly good agreement with traditionally method and the tunnel experimental results. Comparisons with the results of time-dependent simulations are also included.

Index Terms - Field velocity, CFD, acceleration derivative, numerical simulation, unsteady flow.
\end{abstract}

\section{I . Introduction}

Acceleration Derivative, which means the derivative of aerodynamic loads to the rate of angle of attack or yaw, respects of damping characteristic of the plunge motion. Traditionally, the force-oscillation test or numerical simulation can not get the acceleration derivatives directly, but the combined derivatives [1-3].

During the numerical simulation based on CFD, the unsteady solvers are written to incorporate so-called grid velocity, which is used to model unsteady flow via grid movement. The mesh-update technology is needed to update the girds during each time step; this increases the computational expense and memory requirements [4-5].

According to the definition of the acceleration derivative, this paper presented a novel method to obtaining the acceleration derivatives based on the concept of the gird velocity. Compare to the gird-movement method, this method is more effective. The flow girds need not to be updated during each time step, accordingly the computational expense and memory requirements will be reduced greatly.

\section{II . CFD Methodology}

The Euler equations were chosen to study the aerodynamic response of the aircraft to a step change in the angle of attack. The Euler equations were chosen since they can able to handle the possibly strong nonlinear shocks at transonic Mach numbers (as compared to the Potential equation) while being computationally efficient (as compared to the Navier-Stokes equations and the resulting fine mesh). In this study, the effect of viscous forces is expected to be unimportant.

The CFD code uses Van Leer or Roe upwinding with higher-order MUSCL-type limiting on the right-hand side for spatial accuracy. A lower-upper symmetric Gauss-Seidel (LUSGS) implicit operator is used on the left-hand side to increase stability and robustness [6].

\section{III . Field Velocity Approach}

In this study, a new approach, called a field velocity approach, is used to calculate the indicial response to a change in the angle of attack. An impulsive change in the angle of attack can be perceived as an impulsive superposition of a uniform velocity field normal to the free-stream. The magnitude of the normal velocity is determined by the magnitude of the indicial angle-of-attack change. This superposed velocity filed is modeled by modifying the grid time metrics. However, the grid in not actually distorted because of the modified time metrics. This is similar to quasisteady calculations, where grid time metrics are calculated, but the mesh is not actually moved [7]. Mathematically, the field velocity approach can be explained by considering the velocity field $\mathbf{v}$ in the computational domain. It can be written as:

$$
\vec{V}=\left(u-x_{\tau}\right) \vec{i}+\left(v-y_{\tau}\right) \vec{j}+\left(w-z_{\tau}\right) \vec{k}
$$

where $u, v, w$ are the components of the velocity along the coordinate directions and $x_{\tau}, y_{\tau}, z_{\tau}$ are the grid time metrics components. For the flow over a stationary wing these components are zero. Let and indicial change in the angle of attack be represented by velocity $w^{\prime}$ along the $z$ direction. Thus, the velocity field becomes:

$$
\vec{V}=\left(u-x_{\tau}\right) \vec{i}+\left(v-y_{\tau}\right) \vec{j}+\left(w-z_{\tau}+w^{\prime}\right) \vec{k}
$$

The field velocity approach models this change velocity field by modifying the time metrics. The modified time metrics are defined as:

$$
\tilde{x}_{\tau} i+\tilde{y}_{\tau} j+\tilde{z}_{\tau} k=x_{\tau} i+y_{\tau} i+\left(z_{\tau}-w^{\prime}\right) i
$$

This simple velocity correction approach, besides being free of numerical oscillations, also provides a way to determine the response resulting from a pure step change in the angle of attack without any accompanying pitch rate term.

\section{IV . Calculation of the acceleration derivatives}

Integrate the definition of acceleration derivatives and the concept of the field velocity method, assuming that there is a 
step change in angle of attack $\Delta \alpha$ in Fig.1; it can be represented by velocity $w^{\prime}$ along the $z$ direction:

$$
w^{\prime}=\frac{V_{\infty} \sin \Delta \alpha}{\cos \left(\alpha_{0}+\Delta \alpha\right)}
$$

where $\alpha_{0}$ is the initial angle of attack.

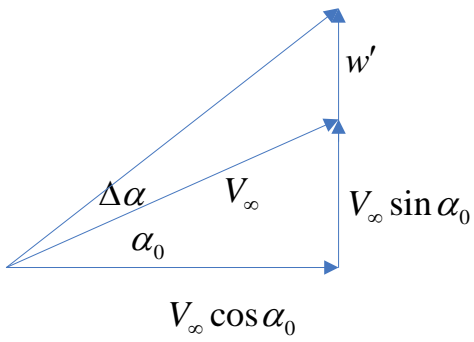

Fig.1 a step change in angle of attack

If the angle of attack changes like this:

$$
\Delta \alpha=\alpha_{m} \sin (k t), \Delta \overline{\dot{\alpha}}=k \alpha_{m} \cos (k t)
$$

According to the equation (5), we can get the unsteady flow of the specified motion. Under the basis of Etkin's unsteady aerodynamic model, the pitching moment coefficient can be written as:

$$
m_{z}=m_{z 0}+m_{z}^{\alpha} \alpha_{m} \sin (k t)+m_{z}^{\bar{\alpha}} k \alpha_{m} \cos (k t)
$$

where $k=\omega l / 2 \mathrm{~V}$ indicates the non-dimensional reduced frequency of the applied motion. The in-phase and out-ofphase components of $m_{z}$, respectively indicated as static derivative $m_{z}^{\alpha}$ and the acceleration derivative $m_{z}^{\bar{\alpha}}$. The dynamic derivative values can be calculated taking the first Fourier coefficients of the time history of $m_{z}$ over one cycle.

$$
\left\{\begin{array}{l}
m_{z}^{\alpha}=\frac{k}{\alpha_{m} \pi} \int_{t}^{t+T}\left(m_{z}-m_{z 0}\right) \cos (k t) d t \\
m_{z}^{\bar{\alpha}}=\frac{1}{\alpha_{m} \pi} \int_{t}^{t+T}\left(m_{z}-m_{z 0}\right) \sin (k t) d t
\end{array}\right.
$$

where $T$ is the period of one cycle of unsteadiness.

\section{V . Example and Results}

Computations are made with basic finner configurations [9]. The basic finner in Fig.2 has been selected as a standard research configuration for validation purposes. The finner has a sharp nose and four wedge shaped fins. The body grid is displayed in Fig. 3.

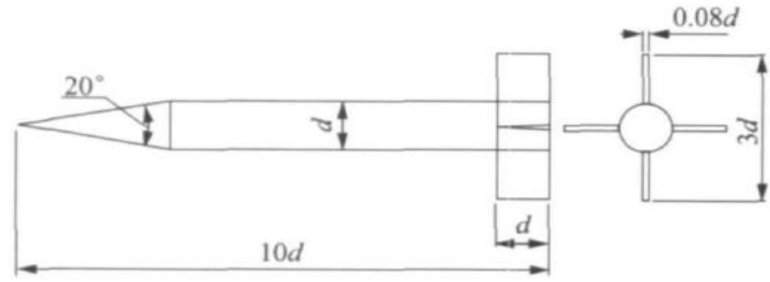

Fig. 2 Basic Finner Model

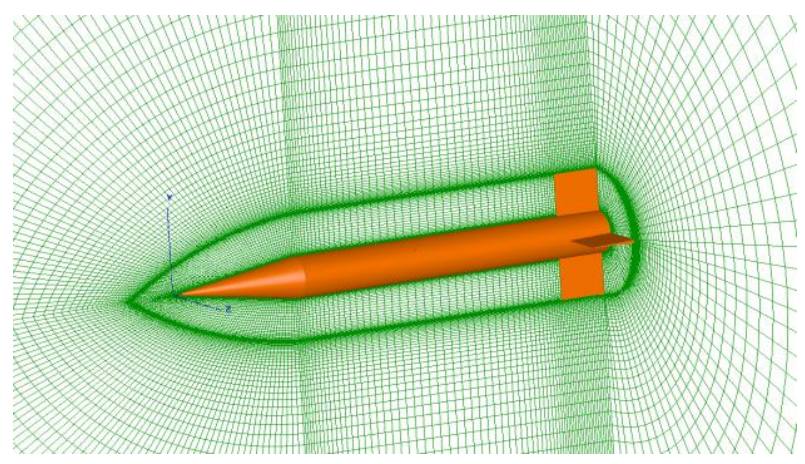

Fig. 3 Computational Grids detail near body

Steady and unsteady computations have been performed for Mach number in $1.5^{\circ}$ angle of attack to determine the static derivative $m_{z}^{\alpha}$ and acceleration derivative $m_{z}^{\bar{\alpha}}$. A constant reduced frequency, $k=0.01$, is used for all Mach numbers. The amplitude is $\alpha_{m}=1^{\circ}$. Two different methods are introduced to calculate the acceleration derivatives; there are grid-movement approach and field velocity approach.

The forced sinusoidal change of the angle of attack results in cyclical histories of the pitching moment $m_{z}$. Figure 4 shows the $m_{z}$ history as a function of attack $\alpha$ for the $\mathrm{C}^{M a=2.5}$. We can see that the field velocity approach results show indeed good agreement with the gird movement approach..
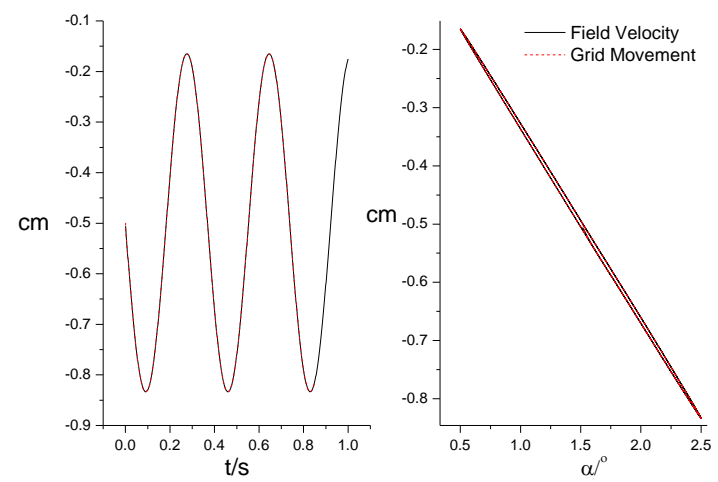

Fig. 4 pitching moment history

Table 1 presents all the results of the derivatives. The experimental data is obtained from Ref.9. The damping derivatives $m_{z}^{\bar{\omega}_{z}}$ equal to the acceleration derivatives subtracted from the combine derivatives, which be obtained form Ref. 4. 
The sign of the acceleration derivative is plus for the $M a=1.58$, it means that the plunging motion is unstable under this Mach number, and also indicates that the sign between damping derivative and acceleration derivative maybe different.

TABLE I Results of Derivatives

\begin{tabular}{|c|c|c|c|c|c|c|}
\hline \multicolumn{2}{|l|}{ Ma } & 1.58 & 1.75 & 1.89 & 2.10 & 2.50 \\
\hline \multirow{3}{*}{ Static derivative } & $\begin{array}{c}\text { Grid } \\
\text { movement }\end{array}$ & -41.0236 & -33.9610 & -29.7153 & -24.9780 & -19.1331 \\
\hline & Field velocity & -41.0254 & -33.9620 & -29.7162 & -24.9784 & -19.1333 \\
\hline & experiment & -42.2853 & -35.0014 & -31.6217 & -24.7692 & -20.7738 \\
\hline \multirow[t]{2}{*}{ Acceleration derivative } & $\begin{array}{c}\text { Grid } \\
\text { movement }\end{array}$ & 2.6848 & -14.0742 & -22.1835 & -28.9742 & -32.3266 \\
\hline & Field velocity & 2.6963 & -14.2431 & -22.1916 & -28.9805 & -32.4077 \\
\hline \multirow[t]{2}{*}{ Damping derivative } & $\begin{array}{c}\text { Grid } \\
\text { movement }\end{array}$ & -462.7634 & -427.3870 & -403.8817 & -374.9710 & -330.5489 \\
\hline & Fieldvelocity & -475.0016 & -437.1490 & -411.8878 & -380.8316 & -334.9547 \\
\hline
\end{tabular}

A comparison of derivatives with mach numbers is displayed in Fig.5. The computed static derivatives are in reasonable agreement with the experiment throughout all selected Mach numbers.

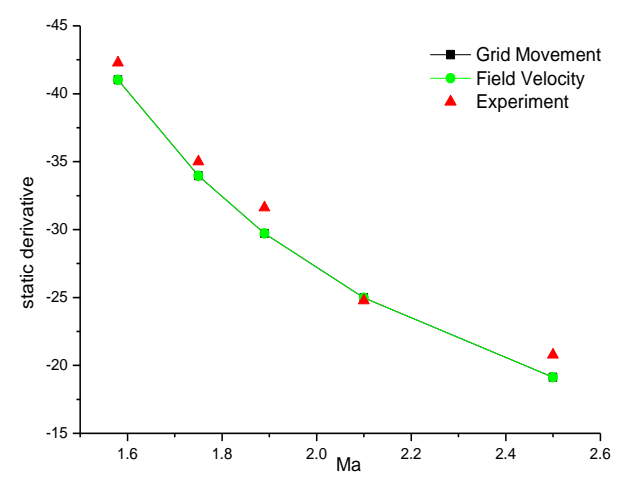

A

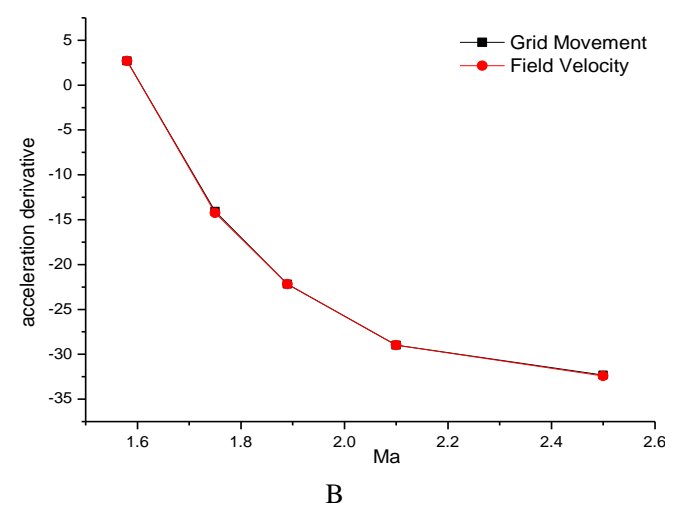

Fig. 5 Variation to derivatives at various Mach numbers

\section{VI . Conclusion}

A novel method of calculating static derivatives and acceleration derivatives using CFD methods has been presented. This method uses field velocity approach to simulate the unsteady flow without updating the field grids during each time step. The results using the presented approach were in good agreement with the experimental data for the basic finner. Another interesting conclusion is that the effect of acceleration derivative may be negative damping, which means the plunging motion is unstable.

\section{Reference}

[1] Yang Enxia. Design for a device of measuring acceleration derivatives of heave oscillation at high swing in low speed wind tunnel. applied science and technology. 2004, 31(12):1-2

[2] Sun Haisheng. A measurement technique for derivatives of aircraft due to acceleration in heave and sideslip at high angle of attack. Experiments and measurements in fluid mechanics. 2001, 15(4):15-19

[3] Liu Wei. Yang Xiaoliang, Zhao Yunfei. Numerical simulation of acceleration derivative of hypersonice aircraft. Acta aerodynamic sinica. 2010, 28(4):426-429

[4] Guo Dong, Xu Min. Chen Shilu. An effective computation method based on field velocity approach for unsteady flow simulation and obtaining dynamic derivatives. Journal of Northwestern Polytechnical University, 2012, 30(5):784-788

[5] Vasudev Parameswaran, James D. Baeder. Indicial aerodynamics in compressible flow-direct computational fluid dynamic calculations. Journal of aircraft, 1997, 34(1):131-133

[6] Yao Weigang. Nonlinear aeroelastic system simulation in time domain[Ph.D], Xi'an: Northwestern Polytechnical University, 2009

[7] Rajneesh Singh, James D.Baeder. Direct Calculation of threedimensional indicial lift response using computational fluid dynamics. Journal of aircraft, 1997, 34(4):465-471

[8] Lomax, H., Indicial aerodynamics. AGARD Manual of aeroelasticity, Pt.II , Nov.1960, Chap.6.

[9] Erdal Oktay, Hasan U.Akay. CFD Predictions of Dynamic Derivatives for missiles. AIAA 2002-0276 